\title{
Operational Modal Identification of Time-Varying Structures via a Vector Multistage Recursive Approach in Hybrid Time and Frequency Domain
}

\author{
Si-Da Zhou, Li Liu, Wu Yang, and Zhi-Sai Ma \\ School of Aerospace Engineering, Beijing Institute of Technology, Zhongguancun South Street 5, Qiushi Building 312, \\ Beijing 100081, China \\ Correspondence should be addressed to Si-Da Zhou; zhousida@bit.edu.cn
}

Received 2 July 2014; Accepted 9 December 2014

Academic Editor: Nuno M. Maia

Copyright (C) 2015 Si-Da Zhou et al. This is an open access article distributed under the Creative Commons Attribution License, which permits unrestricted use, distribution, and reproduction in any medium, provided the original work is properly cited.

\begin{abstract}
Real-time estimation of modal parameters of time-varying structures can conduct an obvious contribution to some specific applications in structural dynamic area, such as health monitoring, damage detection, and vibration control; the recursive algorithm of modal parameter estimation supplies one of fundamentals for acquiring modal parameters in real-time. This paper presents a vector multistage recursive method of modal parameter estimation for time-varying structures in hybrid time and frequency domain, including stages of recursive estimation of time-dependent power spectra, frozen-time modal parameter estimation, recursive modal validation, and continuous-time estimation of modal parameters. An experimental example validates the proposed method finally.
\end{abstract}

\section{Introduction}

In the real world, many engineering structures, such as trafficexcited bridges, launch vehicles with varying fuel mass, airplanes in flight with varying additional aerodynamic effects, deployable and flexible geometry-variable aerospace structures, and rotating machinery, show properties changing with time. In many real-life applications, the excitation on the time-varying structures is unknown and random so that operational or output-only methods are appropriate.

In the past decades, many time-domain parametric approaches of dynamic identification for time-varying structures were presented. Petsounis and Fassois $[1,2]$ presented the time-dependent autoregressive moving average (TARMA) representation for the modeling of nonstationary stochastic vibration. Poulimenos and Fassois [3] surveyed and compared several approaches of TARMA-based nonstationary random vibration modeling including unstructured parameter evolution, stochastic parameter evolution, and deterministic parameter evolution. Poulimenos et al. [4, 5] estimated the modal parameters via the TARMA-based approaches and validated them with a laboratory experiment.
Liu $[6,7]$ proposed a state space-based approach for linear time-varying systems via decomposing a series of Hankel matrices that are assembled by output response data or additional input data with singular value decomposition (SVD). Liu and Deng $[8,9]$ improved the state space-based approach for linear time-varying system through making it less sensitive to noise and validated the identification algorithm with an experiment of a moving cantilever beam.

Meanwhile, several time-frequency analysis-based nonparametric identification approaches were developed in the past decade. Ghanem and Romeo [10] presented a waveletbased identification approach, which transforms the classic governing equation of motion into a wavelet expanded form by projecting the physical responses to a series of wavelet coefficients and identified the modal parameters by solving the expanded-form equation. Roshan-Ghias et al. [11] estimated modal parameters using smoothed pseudo WignerVille distribution (SPWVD), which represents the analytical explicit responses onto WVD plots, and estimated the natural frequency and damping ratio of a SDOF system with tracking the ridge of these plots. Meanwhile, some approaches using Hilbert transform (HT) or Hilbert-Huang transform (HHT) 
were proposed. Xu et al. [12] decomposed the responses into a series of single components with Gabor expansion and identified the modal parameters of these single-component signals with HT.

The batch methods of the model parameter estimation for time-varying structures are overviewed above. Realtime estimation of modal parameters of time-varying can contribute to some specific applications, such as the health monitoring, the damage detection, and the vibration control. Furthermore, the recursive algorithms of modal parameter estimation supply necessary fundamentals for acquiring modal parameters in real-time. Some recursive methods for time-varying systems are also presented in past years. For instance, Lourens et al. [13] developed an augmented Kalman filter based method for force identification in structural dynamics. Song and Pei [14] presented a recursive approach of flutter analysis based on the time-frequency analysis. Some classic recursive algorithms are also introduced in Ljung's book [15].

Moreover, recently the methods of mixing the time domain and frequency domain obtained good results. For instance, Calinoiu et al. [16] proposed a new mixed timeand frequency-domain method for estimating the frequency. In author's previous work, the time-frequency-domain twostage least square method of modal parameter estimation for time-varying structures is presented [17], which can estimate the frequency and mode shapes well.

This paper attempts to present a multistage recursive approach for the potential applications of real-time estimation methods of modal parameters in the hybrid time and frequency domain by choosing and reproducing the available time-domain and frequency-domain methods into the four sequential stages. The reminder of this paper is organized as follows. Section 2 proposes the conceptual design of the multistage recursive estimator. Sections 3 to 6 present the four stages of the proposed estimator, respectively. The proposed method is validated by an experiment in Section 7 .

\section{Design of the Multistage Recursive Estimator}

Different from the common estimators of modal parameters of time-varying structures, the four stages of this proposed recursive estimator in the hybrid time and frequency domain are sequential designed. The basic idea and the procedure of the multistage recursive estimator are shown in Figure 1.

As shown in the first box in Figure 1, when the new responses at the time $t$ are measured, the response vector at the time $t$ is transferred to Stage I estimation. In this stage, the power spectra at the time $t$ are estimated. In addition, the frozen-time modal parameters will be estimated by the frequency-domain method in the next stage, so the selection of the frequency band is possible, which is an advantage of the frequency-domain methods over the time-domain methods. The capability of the frequency band selection favours to achieve the parsimony of the estimation algorithm and may improve the accuracy and quality of the estimation in a limited frequency band.
In Stage II as shown in the second box in Figure 1, the frozen-time modal parameters at time $t$ are estimated by the polyreference least square complex frequency-domain method [18] (pLSCF) based on the power spectra at time $t$ within the interested frequency band. After the estimation of this stage, the modal parameters at time $t$ are obtained, including the physical and mathematical ones, because the order of the parametric model is unknown and a relative high order is given. Therefore, the modal validation is necessary.

For time-invariant structures, the modal parameters are validated by some specific criteria or the stabilization diagram. Some automatic stabilization diagram approaches have been presented [19]. The latter is commonly used in most cases of experimental modal analysis, which is achieved by varying the order of the parametric model and tracking the invariance of the physical modes with the model order. However, these criteria and diagrams are not directly suitable for time-varying structures due to the time variation. The time variation results in difficulty of comparing across multiple time instants and a massive data set due to the varying time instants as well as the varying model orders in terms of using stabilization diagram. Therefore, in Stage III as shown in the third box in Figure 1, the modal parameters at time $t$ estimated in the last stage are validated by the fuzzy clustering in a recursive way. After the validation based on the recursive fuzzy clustering, the modal parameters at time $t$ are automatically ordered by the maximal membership and the quality of the corresponding modal parameters is given by their memberships.

In Stage IV as shown in the fourth box in Figure 1, the continuous-time-varying modal parameters are estimated by a recursive least square approach via using the validated frozen-time modal parameters from the last stage and the memberships of the corresponding modal parameters are used as the weights in the least square estimation.

In the proposed multistage estimator, the response data at the current and past time instants are used and once the response data at time $t$ are measured, the modal parameters at that time instant are estimated. Therefore, this method is recursive, which has the computational parsimony and is potentially suitable for the real-time acquisition of the modal estimation for time-varying structures.

To clarify the workflow of the proposed method through presenting the relations and dataflow among the different stages, Figure 2 shows the detail flowchart of the estimator.

As shown in Figure 2, there are two phases in the estimator for initialization and recursive estimation of the modal parameter estimation. In both phases, the four stages are included, but the purpose of the two phases is partly different. The phase of initialization is to find the prototypes of each mode and to achieve the convergence of Stage I by using a short one-batch data (before the given time instant $t_{I}$ ), in which Stage III is a general fuzzy clustering of the one-batch data. The phase of recursive estimation achieves the recursive estimation of modal parameter, in which the four stages are all recursive and only the data at current time instant are added through an iterative way. 


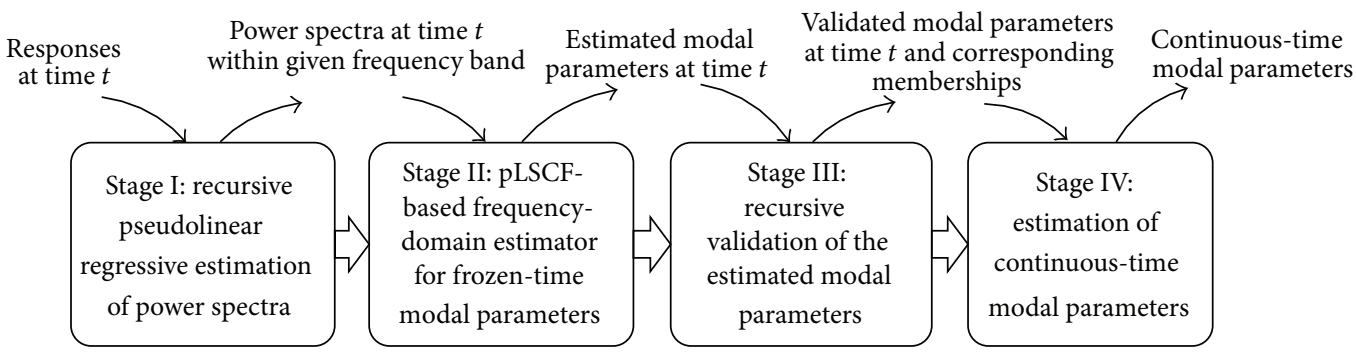

FIGURE 1: Conceptual procedure of the estimator.
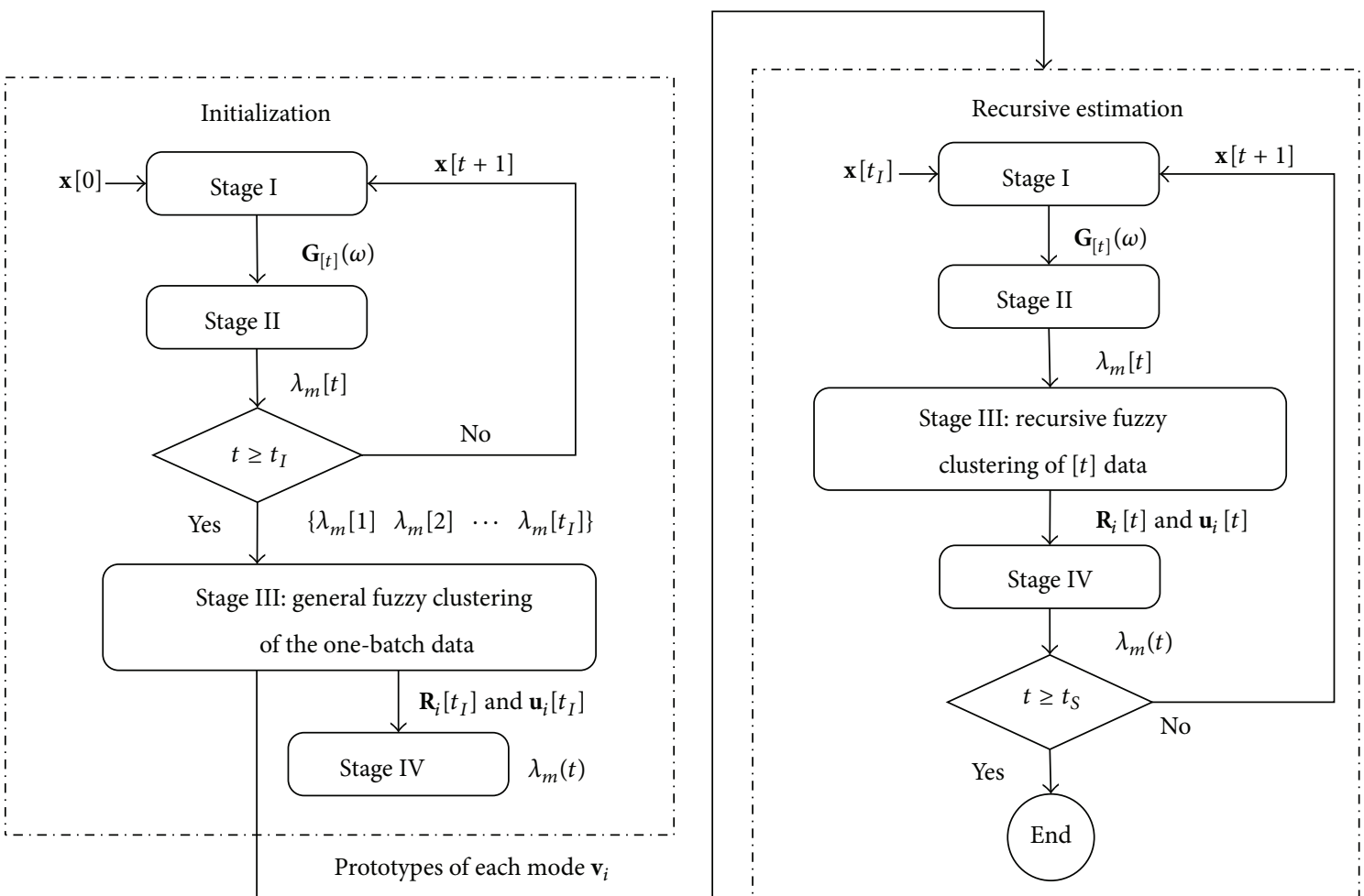

FIgURE 2: Detail flowchart of the estimator.

\section{Stage I: Recursive Pseudolinear Regressive Estimation of Power Spectra}

3.1. Parametric Representation of Responses for TV Structural Systems. For multioutput linear TV structural systems, the multiresponses can be expressed by the forward vector timedependent autoregressive moving average (VTARMA) model as

$$
\begin{array}{r}
\mathbf{x}[t]+\sum_{i=1}^{n_{a}} \mathbf{A}_{i}[t] \mathbf{x}[t-i]=\mathbf{e}[t]+\sum_{i=1}^{n_{c}} \mathbf{C}_{i}[t] \mathbf{e}[t-i], \\
\mathbf{e}[t] \sim \operatorname{NID}(\mathbf{0}, \mathbf{\Sigma}[t]), \quad t=n_{a}+1, \ldots, N,
\end{array}
$$

where $\mathbf{x}[t] \in \mathbb{R}^{N_{o} \times 1}$ is the vector of responses, $\mathbf{A}_{i}[t] \in \mathbb{R}^{N_{o} \times N_{o}}$ and $\mathbf{C}_{i}[t] \in \mathbb{R}^{N_{o} \times N_{o}}$ are the $i$ th autoregressive (AR) and moving average (MA) coefficient matrix, $\mathbf{e}[t] \in \mathbb{R}^{N_{o} \times 1}$ is the uncorrelated residual vector with zero mean and covariance matrix $\Sigma[t] \in \mathbb{R}^{N_{o} \times N_{o}}$, and $n_{a}$ and $n_{c}$ denote the $\mathrm{AR}$ and
MA orders. The AR coefficient matrix and the MA coefficient matrix are defined by

$$
\begin{gathered}
\mathbf{A}_{i}[t]=\left[\begin{array}{cccc}
a_{1,1}^{(i)} & a_{1,2}^{(i)} & \cdots & a_{1, N_{o}}^{(i)} \\
a_{2,1}^{(i)} & a_{2,2}^{(i)} & \cdots & a_{2, N_{o}}^{(i)} \\
\vdots & \vdots & \ddots & \vdots \\
a_{N_{o}, 1}^{(i)} & a_{N_{o}, 2}^{(i)} & \cdots & a_{N_{o}, N_{o}}^{(i)}
\end{array}\right], \\
\mathbf{C}_{i}[t]=\left[\begin{array}{cccc}
c_{1,1}^{(i)} & c_{1,2}^{(i)} & \cdots & c_{1, N_{o}}^{(i)} \\
c_{2,1}^{(i)} & c_{2,2}^{(i)} & \cdots & c_{2, N_{o}}^{(i)} \\
\vdots & \vdots & \ddots & \vdots \\
c_{N_{o}, 1}^{(i)} & c_{N_{o}, 2}^{(i)} & \cdots & c_{N_{o}, N_{o}}^{(i)}
\end{array}\right] .
\end{gathered}
$$


Based on (1) and (2), the VTARMA model is rewritten in a general form as

$$
\mathbf{x}[t]=\boldsymbol{\Phi}^{T}[t] \boldsymbol{\vartheta}[t]+\mathbf{e}[t],
$$

where $\boldsymbol{\vartheta}[t]$ is the vector form of the combined definition of AR and MA coefficients (see (2)) defined by

$\boldsymbol{\vartheta}[t]$

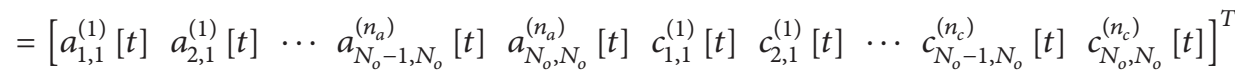

$$
\begin{aligned}
& \in \mathbb{R}^{N_{o}^{2}\left(n_{a}+n_{c}\right) \times 1}
\end{aligned}
$$

and $\Phi[t]$ is defined by

$\Phi[t]$

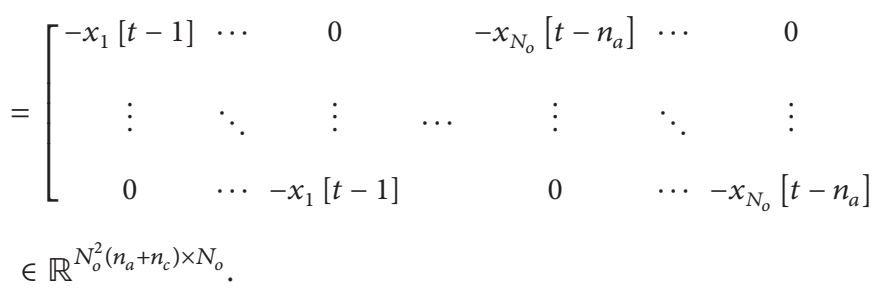

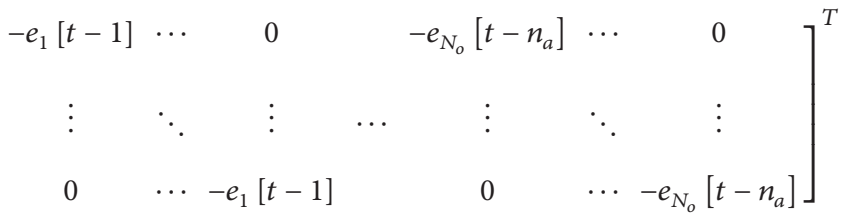

3.2. Recursive Pseudolinear Regression. As shown in (3), the VTARMA model has been represented by a linear equation. However, the predict errors $e_{k}[t-i]$ are not known, so they are approximated by $\widehat{e}_{k}[t, \widehat{\vartheta}[t-i-1]]$, which are obtained at time $t-i$ using the estimated parameters at time $t-i-1$. The response data matrix $\Phi[t]$ does not only depend on the responses but also on the $\boldsymbol{\vartheta}[t-i]$; (3) is actually a pseudolinear equation.

The pseudolinear regression (PLR) for the parameter estimation of the time-invariant systems has been proposed [15] as

$$
\widehat{\boldsymbol{\vartheta}}_{\mathrm{PLR}}=\operatorname{sol}\left\{\frac{1}{N} \sum_{t=1}^{N} \boldsymbol{\Phi}[t, \boldsymbol{\vartheta}]\left(y[t]-\boldsymbol{\Phi}^{T}[t, \boldsymbol{\vartheta}] \boldsymbol{\vartheta}\right)=0\right\},
$$

which means the solution of a nonlinear equation. Therefore, the iterative solution is necessary. In the $(r-1)$ th iteration, a linear equation can be achieved as follows:

$$
\frac{1}{N} \sum_{t=1}^{N} \boldsymbol{\Phi}\left[t, \boldsymbol{\vartheta}_{\mathrm{PLR}}^{\langle r-1\rangle}\right]\left(y[t]-\boldsymbol{\Phi}^{T}\left[t, \boldsymbol{\vartheta}_{\mathrm{PLR}}^{\langle r-1\rangle}\right] \boldsymbol{\vartheta}\right)=0 .
$$

Thus linear equation (7) can be solved by

$$
\begin{aligned}
\boldsymbol{\vartheta}_{\mathrm{PLR}}^{\langle r\rangle}= & \left(\frac{1}{N} \sum_{t=1}^{N} \boldsymbol{\Phi}\left[t, \boldsymbol{\vartheta}_{\mathrm{PLR}}^{\langle r-1\rangle}\right] \boldsymbol{\Phi}^{T}\left[t, \boldsymbol{\vartheta}_{\mathrm{PLR}}^{\langle r-1\rangle}\right]\right)^{-1} \\
& \times\left(\frac{1}{N} \sum_{t=1}^{N} \boldsymbol{\Phi}\left[t, \boldsymbol{\vartheta}_{\mathrm{PLR}}^{\langle r-1\rangle}\right] \mathbf{x}[t]\right)
\end{aligned}
$$

Making one new iteration, at the same time a new measurement is brought in; introducing the forgetting factor $\lambda$ and using the matrix inverse lemma in the community of recursive regression, the recursive pseudolinear regression (RPLR) $[5,15]$ can be conducted as follows.

Parameter estimates update:

$$
\widehat{\boldsymbol{\vartheta}}[t]=\widehat{\boldsymbol{\vartheta}}[t-1]+\mathbf{K}[t] \widehat{\mathbf{e}}[t \mid t-1] .
$$

Prediction errors:

$$
\widehat{\mathbf{e}}[t \mid t-1]=\mathbf{x}[t]-\widehat{\mathbf{x}}[t \mid t-1]=\mathbf{x}[t]-\boldsymbol{\Phi}^{T}[t] \boldsymbol{\vartheta}[t-1] .
$$

Adaptive gain:

$$
\mathbf{K}[t]=\mathbf{P}[t-1] \boldsymbol{\Phi}[t]\left\{\lambda \mathbf{I}_{N_{o}}+\Phi^{T}[t] \mathbf{P}[t-1] \boldsymbol{\Phi}[t]\right\}^{-1} .
$$

“Covariance" update:

$$
\mathbf{P}[t]=\lambda^{-1}\left\{\mathbf{P}[t-1]-\mathbf{K}[t] \boldsymbol{\Phi}^{T}[t] \mathbf{P}[t-1]\right\} .
$$

Different from the original PLR, its recursive version, RPLR, can track the time-variant features of the TV structural systems, which are represented by the time-dependent parameter vector $\mathfrak{\vartheta}[t]$.

3.3. Power Spectra Estimation Based on the Estimates from the $R P L R$. Using the time-dependent parameter vector $\widehat{\boldsymbol{\vartheta}}[t]$ of 
the VTARMA model as estimated above, thus, the frozentime power spectra can be calculated by [20] as follows:

$$
\begin{aligned}
\mathbf{G}_{[t]}^{\mathrm{RPLR}}(\omega)= & \frac{1}{2 \pi}\left(\widehat{\mathbf{A}}\left[e^{-j \omega T_{s}}, t\right]\right)^{-1} \widehat{\mathbf{C}}\left[e^{-j \omega T_{s}}, t\right] \\
& \times \widehat{\boldsymbol{\Sigma}}[t]\left(\left(\widehat{\mathbf{A}}\left[e^{-j \omega T_{s}}, t\right]\right)^{-1} \widehat{\mathbf{C}}\left[e^{-j \omega T_{s}}, t\right]\right)^{H},
\end{aligned}
$$

where $\widehat{\mathbf{A}}\left[e^{-j \omega T_{s}}, t\right]$ and $\widehat{\mathbf{C}}\left[e^{-j \omega T_{s}}, t\right]$ are defined by

$$
\begin{aligned}
& \widehat{\mathbf{A}}\left[e^{-j \omega T_{s}}, t\right]=\mathbf{I}_{N_{o}}+\sum_{i=1}^{n_{a}} \widehat{\mathbf{A}}_{i}[t] e^{-j i \omega T_{s},} \\
& \widehat{\mathbf{C}}\left[e^{-j \omega T_{s}}, t\right]=\mathbf{I}_{N_{o}}+\sum_{i=1}^{n_{c}} \widehat{\mathbf{C}}_{i}[t] e^{-j i \omega T_{s}}
\end{aligned}
$$

and the covariance matrix of the residual vector is estimated by

$$
\widehat{\Sigma}[t]=\frac{1}{2 L+1} \sum_{\tau=t-L}^{t+L} \widehat{\mathbf{e}}[t] \widehat{\mathbf{e}}[t]^{T},
$$

where $\widehat{\mathbf{e}}[t]$ is estimated by $\widehat{\mathbf{e}}[t \mid t-1]$ as shown (10) and $L$ is the length of the smoothing window.

\section{Stage II: pLSCF-Based Frequency-Domain Estimator for Frozen-Time Modal Parameters}

With the white-noise assumption, one of the fundamentals in the community of the operational modal analysis, the power spectra can be expressed by the right matrix fraction model as the same form as the representation of the frequency response functions. Thus, using the power spectra at time instant $t$, the corresponding modal parameters at that time instant can be estimated by the polyreference least square complex frequency-domain [18] (pLSCF) method as below.

The parametric model for estimation in the frequency domain is expressed by

$$
\mathbf{G}_{k}(\omega)=\mathbf{Y}_{k}(\omega) \mathbf{Z}^{-1}(\omega),
$$

where $k=1,2, \ldots, N_{o}$ with the number of outputs $N_{o}$ and the complex-frequency-domain matrices $\mathbf{Y}_{k}(\omega)$ and $\mathbf{Z}(\omega)$ are represented by the matrix polynomials as

$$
\mathbf{Y}_{k}(\omega)=\sum_{j=0}^{n_{p}} \mathbf{Y}_{k, j} \Omega_{j}(\omega) \quad \mathbf{Z}(\omega)=\sum_{j=0}^{n_{p}} \mathbf{Z}_{j} \Omega_{j}(\omega),
$$

where $n_{p}$ is the order of the matrix fraction model and the complex-frequency-domain basis function $\Omega_{j}(\omega)$ is defined as the $z$-domain form as $e^{-j \omega T_{s} j}\left(T_{s}\right.$ is the sampling interval). Thus the unknown parameters in the matrix fraction model can be rewritten as the vector form by

$$
\boldsymbol{\theta}=\left[\begin{array}{c}
\boldsymbol{\beta}_{1} \\
\boldsymbol{\beta}_{2} \\
\vdots \\
\boldsymbol{\beta}_{N_{o} N_{i}} \\
\boldsymbol{\alpha}
\end{array}\right], \quad \text { with } \boldsymbol{\beta}_{k}=\left[\begin{array}{c}
\mathbf{Y}_{k, 0} \\
\mathbf{Y}_{k, 1} \\
\vdots \\
\mathbf{Y}_{k, n_{p}}
\end{array}\right], \boldsymbol{\alpha}=\left[\begin{array}{c}
\mathbf{Z}_{0} \\
\mathbf{Z}_{1} \\
\vdots \\
\mathbf{Z}_{n_{p}}
\end{array}\right]
$$

The cost function of the least square estimator is commonly defined by

$$
\ell_{\mathrm{NLS}}(\boldsymbol{\theta})=\sum_{k=1}^{N_{o}} \sum_{f=1}^{N_{f}}\left|\varepsilon_{k}^{\mathrm{NLS}}\left(\omega_{f}, \boldsymbol{\theta}\right)\right|^{2},
$$

with the frequency sampling at $f=1,2, \ldots, N_{f}$ and the equation errors are

$$
\boldsymbol{\varepsilon}_{k}^{\mathrm{LS}}\left(\omega_{f}, \boldsymbol{\theta}\right)=\mathbf{B}_{k}\left(\omega_{f}, \boldsymbol{\beta}_{k}\right)-\widetilde{\mathbf{G}}_{k}\left(\omega_{f}\right) \mathbf{A}\left(\omega_{f}, \boldsymbol{\alpha}\right),
$$

where $\widetilde{\mathbf{G}}_{k}\left(\omega_{f}\right)$ are the measured spectra.

The matrix form of (20) can be rewritten by

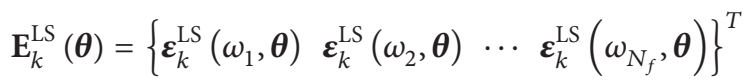

$$
\begin{aligned}
& =\left[\begin{array}{ll}
\Theta_{k} & \boldsymbol{\Xi}_{k}
\end{array}\right]\left\{\begin{array}{c}
\boldsymbol{\beta}_{k} \\
\boldsymbol{\alpha}
\end{array}\right\},
\end{aligned}
$$

with

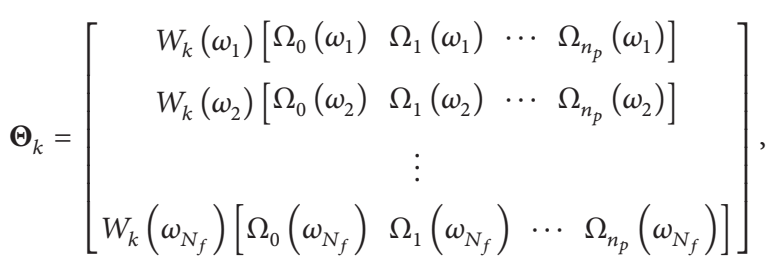

$\Xi_{k}$

$$
=\left[\begin{array}{cccc}
-W_{k}\left(\omega_{1}\right)\left[\Omega_{0}\left(\omega_{1}\right)\right. & \Omega_{1}\left(\omega_{1}\right) & \cdots & \left.\Omega_{n_{p}}\left(\omega_{1}\right)\right] \otimes \widetilde{\mathbf{G}}_{k}\left(\omega_{1}\right) \\
-W_{k}\left(\omega_{2}\right)\left[\Omega_{0}\left(\omega_{2}\right)\right. & \Omega_{1}\left(\omega_{2}\right) & \cdots & \left.\Omega_{n_{p}}\left(\omega_{2}\right)\right] \otimes \widetilde{\mathbf{G}}_{k}\left(\omega_{2}\right) \\
\vdots & & \\
-W_{k}\left(\omega_{N_{f}}\right)\left[\Omega_{0}\left(\omega_{N_{f}}\right)\right. & \Omega_{1}\left(\omega_{N_{f}}\right) & \cdots & \left.\Omega_{n_{p}}\left(\omega_{N_{f}}\right)\right] \otimes \widetilde{\mathbf{G}}_{k}\left(\omega_{N_{f}}\right)
\end{array}\right] .
$$

Thus, the cost function as shown in (19) can be rewritten as

$$
\ell_{\mathrm{LS}}(\boldsymbol{\theta})=\sum_{k=1}^{N_{o}}\left[\begin{array}{ll}
\boldsymbol{\beta}_{k}^{H} & \boldsymbol{\alpha}^{H}
\end{array}\right]\left[\begin{array}{cc}
\mathbf{P}_{k} & \mathbf{Q}_{k} \\
\mathbf{Q}_{k}^{H} & \mathbf{R}_{k}
\end{array}\right]\left[\begin{array}{c}
\boldsymbol{\beta}_{k} \\
\boldsymbol{\alpha}
\end{array}\right]
$$

where $\mathbf{P}_{k}=\operatorname{Re}\left(\boldsymbol{\Theta}_{k}^{H} \boldsymbol{\Theta}_{k}\right), \mathbf{Q}_{k}=\operatorname{Re}\left(\boldsymbol{\Theta}_{k}^{H} \boldsymbol{\Xi}_{k}\right)$, and $\mathbf{R}_{k}=\operatorname{Re}\left(\boldsymbol{\Xi}_{k}^{H} \boldsymbol{\Xi}_{k}\right)$.

When minimizing the cost function as (23), the normal equation of the least squares is achieved as

$$
\begin{gathered}
\frac{\partial \ell_{\mathrm{LS}}(\boldsymbol{\theta})}{\partial \boldsymbol{\beta}_{k}}=2\left(\mathbf{P}_{k} \boldsymbol{\beta}_{k}+\mathbf{Q}_{k} \boldsymbol{\alpha}\right)=0, \quad k=1, \ldots, N_{o}, \\
\frac{\partial \ell_{\mathrm{LS}}(\boldsymbol{\theta})}{\partial \boldsymbol{\alpha}}=2\left[\sum_{k=1}^{N_{o}}\left(\mathbf{Q}_{k}^{H} \boldsymbol{\beta}_{k}+\mathbf{R}_{k} \boldsymbol{\alpha}\right)\right]=0 .
\end{gathered}
$$

Substituting (24) into (25), the reduced normal equation is obtained as

$$
2\left[\sum_{k=1}^{N_{o}}\left(\mathbf{R}_{k}-\mathbf{Q}_{k}^{H} \mathbf{P}_{k}^{-1} \mathbf{Q}_{k}\right)\right] \boldsymbol{\alpha}=\mathbf{D} \boldsymbol{\alpha}=0 .
$$


Furthermore, giving the appropriate constraint on the $\boldsymbol{\alpha}$, its nontrivial solution can be conducted by referring to [21] and $\boldsymbol{\beta}_{k}$ can be calculated by back-substituting $\boldsymbol{\alpha}$ into (24). The computational complexity of this stage is approximately $O\left(2 N_{o} N_{r} n_{p}^{2} N_{f}\right)$ with the $N_{r}$ reference.

After the unknown parameter vector is achieved, the modal parameter, including the modal frequency, damping ratio, and the operational reference vector can be obtained by solving a general right eigenvalue problem as

$$
\left(\boldsymbol{\Gamma}-\lambda_{m} \mathbf{I}\right) \mathbf{V}_{m}=0,
$$

with

$$
\begin{gathered}
\boldsymbol{\Gamma}=\left[\begin{array}{cccc}
\mathbf{A}_{n_{p}-1}^{\prime} & \cdots & \mathbf{A}_{1}^{\prime} & \mathbf{A}_{0}^{\prime} \\
\mathbf{I} & \cdots & 0 & 0 \\
\vdots & \mathbf{I} & \ddots & 0 \\
0 & 0 & \mathbf{I} & 0
\end{array}\right] \text { with } \mathbf{A}_{i}^{\prime}=-\mathbf{A}_{n_{p}}^{-1} \mathbf{A}_{i}, \\
\mathbf{V}_{m}=\left[\begin{array}{c}
\lambda_{m}^{n-1} \mathbf{L}_{m} \\
\vdots \\
\lambda_{m} \mathbf{L}_{m} \\
\mathbf{L}_{m}
\end{array}\right],
\end{gathered}
$$

where $\mathbf{L}_{m}$ are the operational reference vector. The modal frequency and damping ratio are contained in the poles $\lambda_{m}$.

Using the pLSCF method as presented above, the modal parameters at the new brought-in time instant can be estimated.

\section{Stage III: Recursive Validation of the Estimated Modal Parameters}

After obtaining the modal parameters at the new brought-in time instant, the validation and order determination of modal parameters are necessary. In this stage, the modal parameters are validated and ordered recursively by using a recursive fuzzy clustering.

5.1. Fuzzy Clustering. Fuzzy cluster analysis or fuzzy clustering partition is a set of data into $C$ clusters and each cluster is represented by its center, also called prototype. In other words, the aim of the fuzzy cluster analysis is to determine the prototypes and the memberships.

Let $\mathbf{X}=\left\{\mathbf{x}_{1}, \mathbf{x}_{2}, \ldots, \mathbf{x}_{N}\right\} \subset \mathbf{R}^{p}$ be a data set with $N \in \mathbf{N}^{1}$ data points. The memberships can be represented by a matrix $\mathbf{U} \in \mathbf{R}^{C \times N}$ with $C \in \mathbf{N}^{1}$ clusters. An element in the membership matrix $u_{i j}$, with $i=1,2, \ldots, C$ and $j=1,2, \ldots, N$, denotes the degree of the $j$ th data point belonging to the $i$ th cluster relative to other clusters. Because the membership matrix, $\mathbf{U}$, represents a probabilistic cluster partition, there are a few constraints on $\mathbf{U}$ :

$$
\begin{gathered}
\forall i \in \mathbf{N}_{[1, C]}^{1}, \quad j \in \mathbf{N}_{[1, N]}^{1}: u_{i j} \in[0,1], \\
\forall j \in \mathbf{N}_{[1, N]}^{1}: \sum_{i=1}^{C} u_{i j}=1, \\
\forall i \in \mathbf{N}_{[1, C]}^{1}: 0<\sum_{j=1}^{N} u_{i j}<N .
\end{gathered}
$$

Equations (29) and (30) imply that the total membership for the $j$ th data point to all $C$ clusters is 1 and, for each cluster, at least one data point belongs to that cluster with a nonzero membership, respectively.

Let $\mathbf{V}=\left\{\mathbf{v}_{1}, \mathbf{v}_{2}, \ldots, \mathbf{v}_{C}\right\} \subset \mathbf{R}^{p}$ be a set of prototypes with $C$ cluster prototypes. $\mathbf{v}_{i}$ is the prototype of the $i$ th cluster.

The fuzzy $c$-means (FCM) [22] algorithm is one of the objective-based fuzzy clustering algorithms. Given the data set $\mathbf{X}$, the membership matrix $\mathbf{U}$, the cluster prototypes $\mathbf{V}$, and the distance function $d$, a scalar objective function is described by

$$
J(\mathbf{X}, \mathbf{U}, \mathbf{V})=\sum_{i=1}^{C} \sum_{j=1}^{N} u_{i j}^{m} d^{2}\left(\mathbf{x}_{j}, \mathbf{v}_{i}\right),
$$

where $J \in \mathbf{R}^{1}$, the exponent $m \in \mathbf{R}_{>1}^{1}$ is the fuzzy exponent, and $d(\cdot, \cdot) \in \mathbf{R}^{1}$ denotes the distance between two vectors, which does not depend on the memberships. Commonly, $m=2$ and $d(\cdot, \cdot)$ is the Euclidean distance function as

$$
\forall i \in \mathbf{N}_{[1, C]}^{1}, j \in \mathbf{N}_{[1, N]}^{1}: d_{i j}^{2}=d^{2}\left(\mathbf{x}_{j}, \mathbf{v}_{i}\right)=\left(\mathbf{x}_{j}-\mathbf{v}_{i}\right)^{T}\left(\mathbf{x}_{j}-\mathbf{v}_{i}\right)
$$

The fuzzy $c$-means algorithm (FCM) is one of the most popular algorithms of fuzzy cluster analysis. There are five phases in FCM.

Phase I. Choosing the number of clusters $C$, the fuzzy exponent $m$, the convergence criterion $\zeta$, and the maximum number of iterations $M$, the initial membership matrix $\mathbf{U}^{(0)}$ is defined randomly, which satisfies the constraints as (29), (30), and (31).

Phase II. Update the cluster prototypes, $\mathbf{V}^{(r)}$, at the $r$ th iteration by

$$
\forall i \in \mathbf{N}_{[1, C]}^{1}: \mathbf{v}_{i}^{(r)}=\frac{\sum_{j=1}^{N}\left(u_{i j}^{(r-1)}\right)^{m} \mathbf{x}_{j}}{\sum_{j=1}^{N}\left(u_{i j}^{(r-1)}\right)^{m}}
$$

Phase III. Calculate the distances $d_{i j}^{(r)}$ for all $i=1,2, \ldots, C$ and $j=1,2, \ldots, N$ using the updated cluster prototypes, $\mathbf{V}^{(r)}$, based on the distance function as defined in (33) or other distance functions.

Phase IV. Update the membership matrix, $\mathbf{U}^{(r)}$, at the $r$ th iteration by

$$
\begin{aligned}
& \forall i \in \mathbf{N}_{[1, C]}^{1}, j \in \mathbf{N}_{[1, N]}^{1}: u_{i j}^{(r)} \\
& = \begin{cases}\frac{1}{\sum_{r=1}^{C}\left(d_{i j}^{(r)} / d_{r j}^{(r)}\right)^{2 /(m-1)}}, & \text { for } \boldsymbol{\Gamma}_{j}=\emptyset \\
\sum_{r \in \Gamma_{j}} u_{r j}^{(r)}=1, & \text { for } \boldsymbol{\Gamma}_{j} \neq \emptyset, j \in \boldsymbol{\Gamma}_{j} \\
0, & \text { for } \boldsymbol{\Gamma}_{j} \neq \emptyset, j \notin \boldsymbol{\Gamma}_{j},\end{cases}
\end{aligned}
$$


where $\emptyset$ is the null set and $\boldsymbol{\Gamma}_{j}=\left\{i \mid d_{i j}^{(r)}=0\right\}$. If $\boldsymbol{\Gamma}_{j}$ contains more than one element, $u_{r j}^{(r)}$ for $r \in \Gamma_{j}$ is not uniquely determined and more operations are needed [22].

Phase $V$. Check the termination criteria including the convergence criterion $\zeta$ and the maximum number of iterations $M$. If $\left\|\mathbf{U}^{(r)}-\mathbf{U}^{(r-1)}\right\|<\zeta$ or $k>M$, the iteration terminates; otherwise, repeat Phase II, Phase III, and Phase IV.

5.2. Recursive Fuzzy Clustering. When the observed data changes with the time, the recursive clustering is necessary to capture the current features of the new-coming data. An approach of recursive fuzzy clustering is introduced as follows [23].

The $i$ th cluster prototype at the time instant $n$ is defined by $\mathbf{v}_{i}(n)$. The relation between the old cluster prototype and a new one can be expressed by

$$
\mathbf{v}_{i}(n+1)=\mathbf{v}_{i}(n)+\Delta \mathbf{v}_{i}(n+1),
$$

with the following increment:

$$
\Delta \mathbf{v}_{i}(n+1)=\frac{u_{i(r+1)}^{m}\left(\mathbf{x}(n+1)-\mathbf{v}_{i}(n)\right)}{\sum_{k=1}^{n} u_{i k}^{m}+u_{i(n+1)}^{m}} .
$$

As shown in (37), the calculation of the membership at the $(n+1)$ th time instant requires the past $n$ memberships, which is against the recursive idea. Therefore, an approximate calculation of the denominator of (37) is achieved by adding the forgetting factors for the past memberships defined as follows:

$$
\mathbf{s}_{i}(n+1)=\beta \mathbf{s}_{i}(n)+u_{i(n+1)}^{m},
$$

where $\beta$ is the forgetting factor and $0<\beta \leq 1$. As shown in (38), the denominator at the time instant $n, \mathbf{s}_{i}(n+1)$, can be calculated by a recursive approach. Furthermore, the current membership is defined by

$$
u_{i(r+1)}=\left(d_{i(n+1)}^{2} \sum_{r=1}^{C}\left(\frac{1}{d_{r(n+1)}^{2}}\right)^{1 /(m-1)}\right)^{-1} .
$$

As presented above, the algorithm is recursive and no convergence condition is required.

5.3. Recursive Fuzzy Validation of the Modal Parameters. Furthermore, the memberships will be considered as the weights in the next continuous-time estimation of modal parameters using a recursive least square approach. The validated modal parameters are expressed into the four subpartitions with the corresponding memberships by

$$
\begin{aligned}
\forall i \in \mathbb{N}_{[1, C]}^{1}, \quad \forall j \in \mathbb{N}_{\left[1, N_{i}\right]}^{1}, \quad N_{i}=\left|\mathbf{R}_{i}[t]\right|: \\
\mathbf{R}_{i}[t]=\left\{\mathbf{x}_{i, 1}, \mathbf{x}_{i, 2}, \ldots, \mathbf{x}_{i, j}, \ldots, \mathbf{x}_{i, N_{i}}\right\}, \\
\mathbf{u}_{i}[t]=\left\{u_{i, 1}, u_{i, 2}, \ldots, u_{i, j}, \ldots, u_{i, N_{i}}\right\},
\end{aligned}
$$

where $\mathbf{x}_{i, j}$ is general data point, which can be the modal frequency or the damping ratio, $u_{i, j}$ is the corresponding membership, and $N_{i}$ is the length of the $i$ th subpartition including the data of time $t$ and before $t$, which is updated with process of recursive estimation.

\section{Stage IV: Estimation of Continuous-Time Modal Parameters}

In this stage, the continuous-time-represented modal parameters are estimated by a recursive least square approach based on the validated modal parameters of time $t$ and before $t$. The cost function of this recursive least squares is defined by

$$
\ell_{i}^{\mathrm{WLS}}\left(\boldsymbol{\gamma}_{i}\right)=\sum_{j=1}^{N_{i}}\left|\varepsilon_{i}^{\mathrm{WLS}}\left(t_{j}, \gamma_{i}\right)\right|^{2},
$$

where $i, j$, and $N_{i}$ are defined in (40) and with the following equation error:

$$
\varepsilon_{i}^{\mathrm{LS}}\left(t_{j}, \gamma_{i}\right)=w_{i, j}\left(x_{i}\left(t_{j}\right)-x_{i, j}\right),
$$

where $w_{i, j}$ are the weights and defined by $w_{i, j}=u_{i, j}^{\kappa}(\kappa$ is the exponent for weighting), $x_{i, j}$ is an arbitrary component in $\mathbf{x}_{i, j}$ and the predict function $x_{i}\left(t_{j}\right)$ is expressed by a polynomial as

$$
x_{i}(t)=\sum_{k=1}^{L} \gamma_{i, k} p_{k}(t)
$$

where $\gamma_{i}=\left\{\gamma_{i, k} \mid k \in \mathbb{N}_{[1, L]}^{1}\right\}$ and the basis function $p_{k}(t)$ are polynomials.

The estimation of the projection coefficients $\gamma_{i}$ can be obtained by minimizing the cost function, (41), as

$$
\widehat{\gamma}_{i}=\arg \min \left(\ell_{i}^{\mathrm{WLS}}\left(\gamma_{i}\right)\right),
$$

where "arg min" means the "argument minimization."

According to (42) and (43), the equation errors can be rewritten by

$$
\begin{aligned}
& \boldsymbol{\varepsilon}_{i}^{\mathrm{WLS}}=\left[\begin{array}{cccc}
w_{i, 1}\left[p_{i, 1}\left(t_{1}\right)\right. & p_{i, 2}\left(t_{1}\right) & \cdots & \left.p_{i, L}\left(t_{1}\right)\right] \\
w_{i, 2}\left[p_{i, 1}\left(t_{2}\right)\right. & p_{i, 2}\left(t_{2}\right) & \cdots & \left.p_{i, L}\left(t_{2}\right)\right] \\
\vdots & & \\
w_{i, N_{i}}\left[p_{i, 1}\left(t_{N_{i}}\right)\right. & p_{i, 2}\left(t_{N_{i}}\right) & \cdots & \left.p_{i, L}\left(t_{N_{i}}\right)\right]
\end{array}\right] \\
& \times\left\{\begin{array}{c}
\theta_{i, 1} \\
\theta_{i, 2} \\
\vdots \\
\theta_{i, L}
\end{array}\right\}-\left\{\begin{array}{c}
w_{i, 1} x_{i, 1} \\
w_{i, 2} x_{i, 2} \\
\vdots \\
w_{i, N_{i}} x_{i, N_{i}}
\end{array}\right\}=\Pi_{i} \gamma_{i}-\Delta_{i} .
\end{aligned}
$$

As defined in (45), the least square problem is linear. Hence, the projection coefficients, $\gamma_{i}$, can be estimated [24] as follows:

$$
\widehat{\gamma}_{i}=\left(\boldsymbol{\Pi}_{i}^{T} \boldsymbol{\Pi}_{i}\right)^{-1} \boldsymbol{\Pi}_{i}^{T} \boldsymbol{\Delta}_{i} .
$$




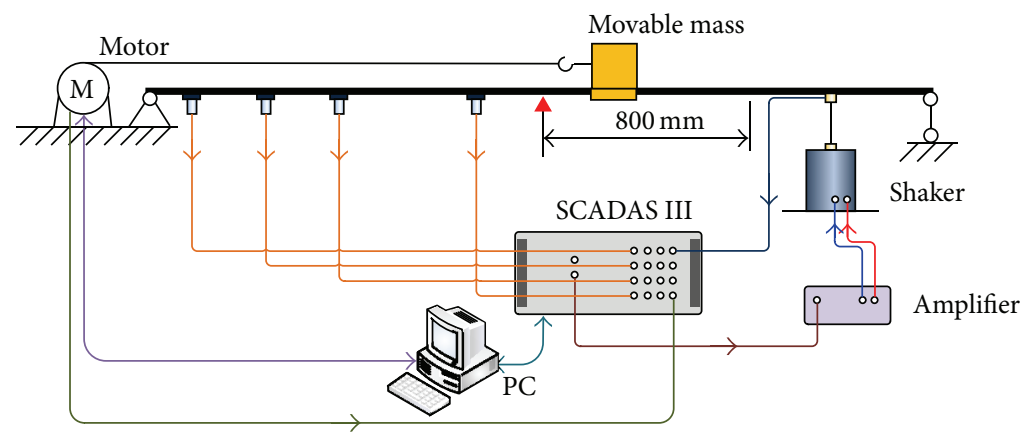

(a)

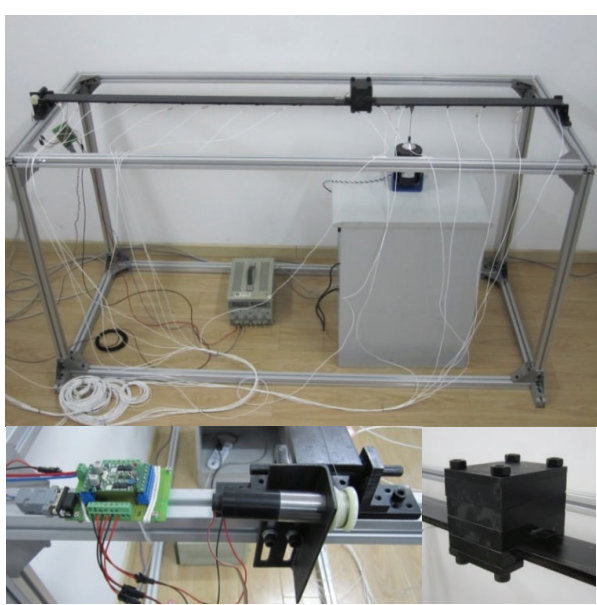

(b)

FiguRE 3: Schematic diagram (a) and real configuration (b) of the experimental structure and its laboratory set-up including the close-up views of the motor and its controller as well as the movable mass.

The $\widehat{\gamma}_{i}$ can be estimated as a recursive behavior. Define the projection coefficients of the $t$ th time instant $\widehat{\gamma}_{i}(t)$; the $\widehat{\boldsymbol{\theta}}_{i}(t+1)$ can be calculated by

$$
\begin{aligned}
\widehat{\gamma}_{t+1} & =\left(\boldsymbol{\Pi}_{t+1}^{T} \boldsymbol{\Pi}_{t+1}\right)^{-1} \boldsymbol{\Pi}_{t+1}^{T} \Delta_{t+1} \\
& =\left(\boldsymbol{\Pi}_{t}^{T} \boldsymbol{\Pi}_{t}+\mathbf{a}_{t+1}^{T} \mathbf{a}_{t+1}\right)^{-1}\left(\boldsymbol{\Pi}_{t}^{T} \Delta_{t}+\mathbf{a}_{t+1}^{T} b_{t+1}\right),
\end{aligned}
$$

with

$$
\begin{aligned}
& \boldsymbol{\Pi}_{t+1}=\left[\begin{array}{c}
\Pi_{t} \\
\mathbf{a}_{t+1}
\end{array}\right], \quad \Delta_{t+1}=\left[\begin{array}{c}
\Delta_{t} \\
b_{t+1}
\end{array}\right], \\
& \mathbf{a}_{t+1}=w_{t+1}\left[\begin{array}{llll}
p_{1}\left(t_{t+1}\right) & p_{2}\left(t_{t+1}\right) & \cdots & p_{L}\left(t_{t+1}\right)
\end{array}\right], \\
& b_{t+1}=w_{t+1} x_{t+1} .
\end{aligned}
$$

\section{Experiment}

In this section, experimental results validate the proposed multistage recursive estimator. The reference modal parameters are obtained by identifying a series of "frozen-configuration" structures of corresponding to the time-varying structure.

7.1. The Structure and the Laboratory Setup. Figure 3 shows the schematic diagram of the experimental structure and its laboratory setup. The structure consists of a steel beam and a steel mass, of which the parameters are listed in Table 1. The beam is simply supported at the two ends by a heavy frame. The mass can slide along the axial direction of the beam driven by a controllable motor from the center to $800 \mathrm{~mm}$ away. The velocity of the mass is constantly $100 \mathrm{~mm} / \mathrm{s}$ under a feedback control of the motor. Eleven piezoelectric accelerometers (sensitivity: $\approx 100 \mathrm{mV} / \mathrm{g}$ ) measure the acceleration responses of the beam at eleven uniformly distributed positions along the axial direction of the beam, although only four are shown in Figure 3(a). A LMS SCADAS III
TABLE 1: Basic parameters of the structure.

\begin{tabular}{lcccc}
\hline & Length $(\mathrm{mm})$ & Width $(\mathrm{mm})$ & Height $(\mathrm{mm})$ & Weight $(\mathrm{kg})$ \\
\hline Beam & 2000 & 60 & 10 & 9.420 \\
Mass & 80 & 100 & 94 & 4.866 \\
\hline
\end{tabular}

system acquires the acceleration response signals and the force signal of the shaker. The PC records the input signals via the SCADAS system and LMS Test.Lab software and starts the motor. The measurement is triggered by the signal from the motor shown as the green arrowed connection in Figure 3(a).

Figure 3(b) shows the real configuration of the structure and the laboratory setup. For the measurement of the baseline models, the input force is measured by an impedance head (sensitivity: $2.248 \mathrm{~V} / \mathrm{kN}$ ). For the measurement of the timevarying case, the input force is assumed as unknown.

7.2. Baseline Parametric Identification of the Fixed-Configuration Structure via the General Time-Invariant Approach. The time-varying structure in this paper can also be considered as a linear parameter-varying (LPV) structure. The structural dynamic characteristics are functions of the position of the mass. While other parameters are fixed, the position of the mass can be continuously time-varying. In other words, when the mass stays at a fixed position, the structural dynamic characteristics, such as modal parameters, can be obtained by the available LTI system identification approaches, which are the "frozen-time" or frozen-configuration reference for the time-varying case [4].

The frozen-configuration structure is the baseline model for the time-varying structure. Moving the mass from the center of the beam to $800 \mathrm{~mm}$ away with a spacing of $10 \mathrm{~mm}$, 81 baseline models can be obtained. The eleven acceleration signals and the force signal are measured with a sampling frequency of $512 \mathrm{~Hz}$ and record length of $2 \mathrm{~s}$. Figure 4 shows the averaged FRFs (the bandwidth is $0-160 \mathrm{~Hz}$, the average 


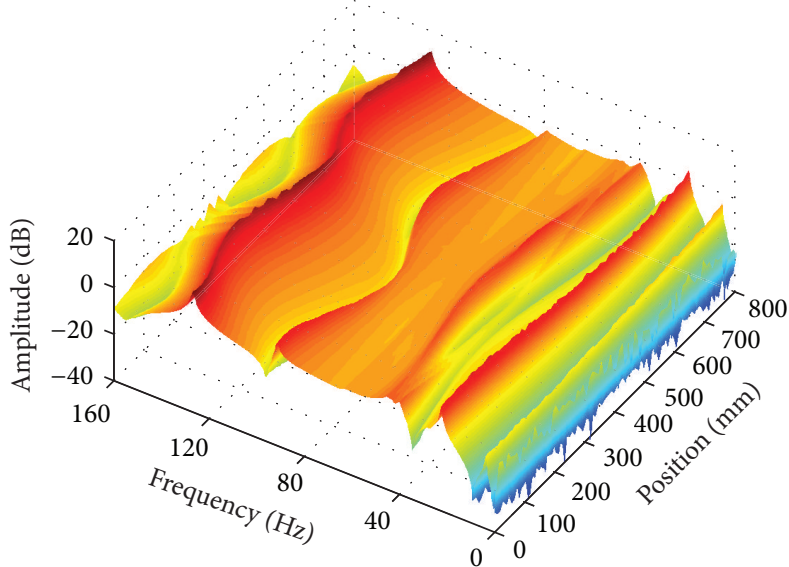

(a)

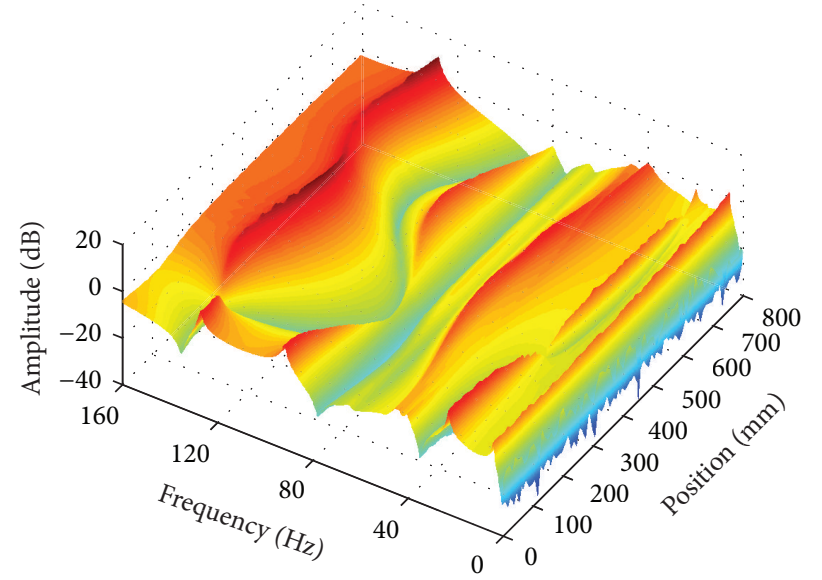

(c)

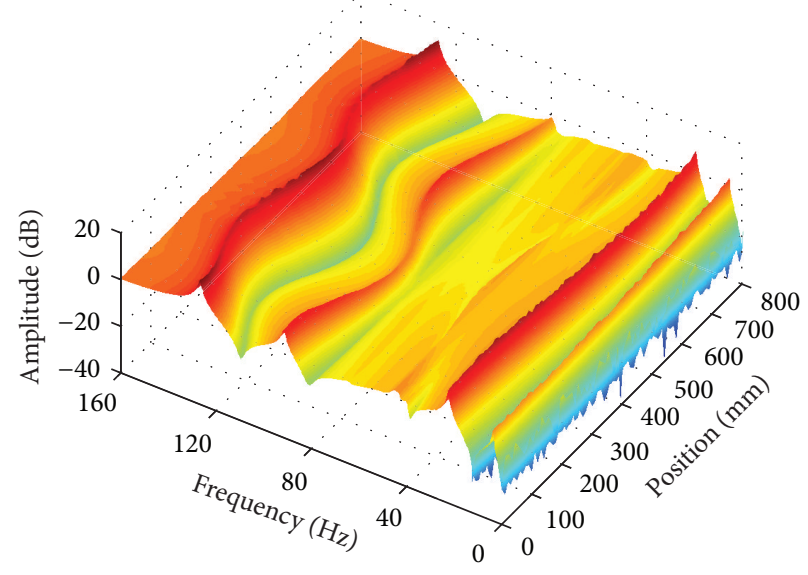

(b)

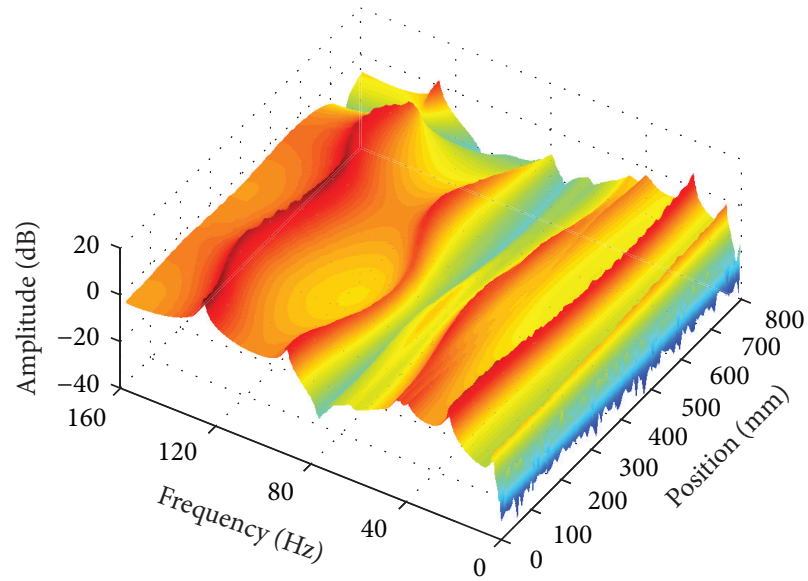

(d)

FIGURE 4: FRFs of the baseline models: (a) $H_{\mathrm{ex}, 1}$, (b) $H_{\mathrm{ex}, 4}$ (c) $H_{\mathrm{ex}, 7}$, and (d) $H_{\mathrm{ex}, 11}$.

number is 5, and the FRFs are estimated by $\mathrm{H} 1$ method [25]) between the excitation and Outputs $1,4,7$, and 11 for the 81 baseline models.

In the FRFs shown in Figure 4, there are five peaks with respect to frequency, which indicate the five modes in the bandwidth $(0-160 \mathrm{~Hz})$.

In order to identify the modal parameters of the baseline models, the least squares complex exponential method (LSCE) [25] is used. Figures 5(a) and 5(b) show the modal frequency and damping ratio of the baseline models, respectively.

7.3. Time-Varying Testing. In this section, the structure is time-varying due to the mass sliding continuously. The setup is the same as that for the "fixed-configuration" structure. The sampling frequency is $512 \mathrm{~Hz}$ and the record length is $8 \mathrm{~s}$. For the sake of comparison between the estimation results of the time-varying structure and the reference values of the baseline models, the relationship between the position of the mass and the time variable is achieved by using the known constant velocity of the mass.
The excitation generated by the shaker is Gaussian white noise. The acceleration is measured at 11 uniformly distributed locations, which forms the basic data set for the modal parameter estimation of the time-varying structure.

Considering the uniformity, Figures 6(a), 6(b), 6(c), and $6(\mathrm{~d})$ show the responses of Outputs $1,4,7$, and 11 of the 11 outputs.

7.4. Recursive Pseudolinear Regressive Estimation of Power Spectra. Once the response data are measured, the power spectra at time $t$ can be obtained. For the sake of presenting the results as the sequence of the stages, the whole batch of the results is shown in the following illustrations rather than as the sequence of the time instant by time instant, although the estimation is recursively processed by time.

Assume that the interested frequency band is from $70 \mathrm{~Hz}$ to $150 \mathrm{~Hz}$; the cross power spectra for Outputs $1,4,7$, and 11 referred to Output 7 are shown in Figure 7.

7.5. pLSCF-Based Frequency-Domain Estimator for FrozenTime Modal Parameters. Based on the pLSCF estimator in 


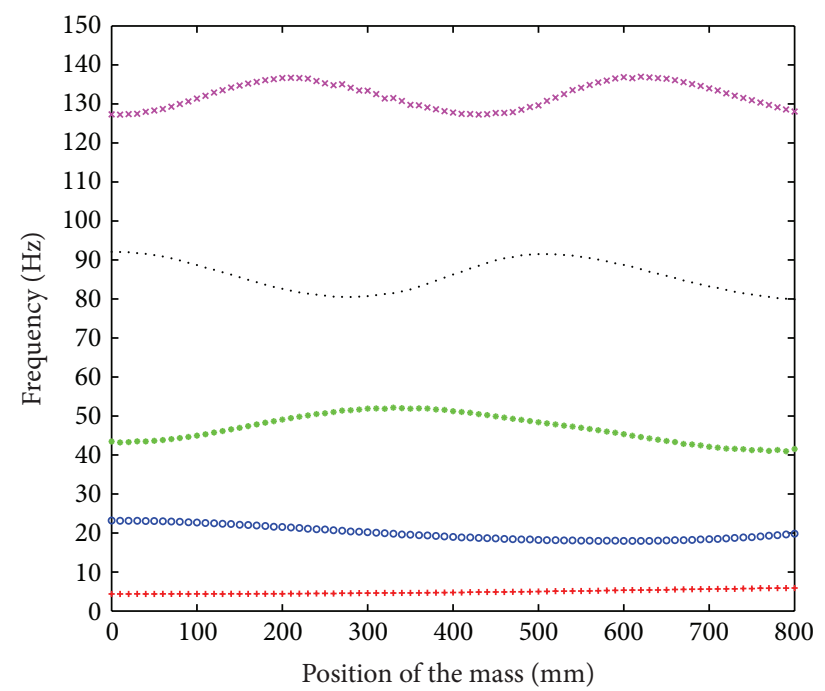

(a)

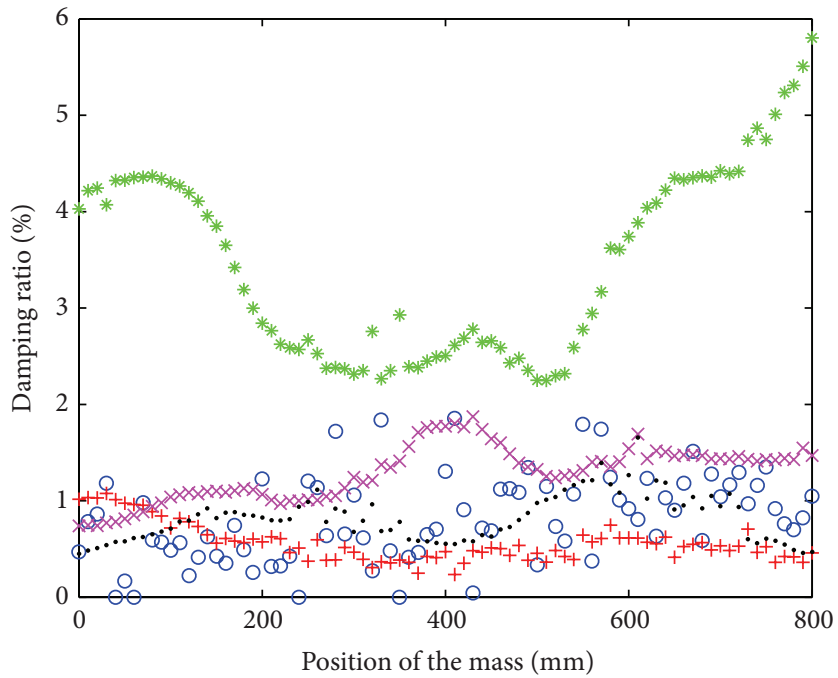

(b)

Figure 5: (a) Modal frequency. (b) Damping ratio for Mode 1 (“•”), Mode 2 (“×”), Mode 3 (“*”), Mode 4 (“+”), and Mode 5 (“O”).

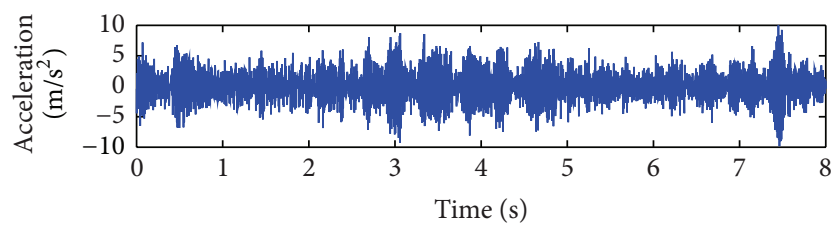

(a)

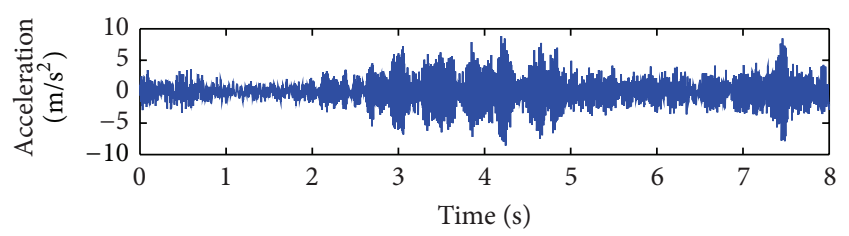

(c)

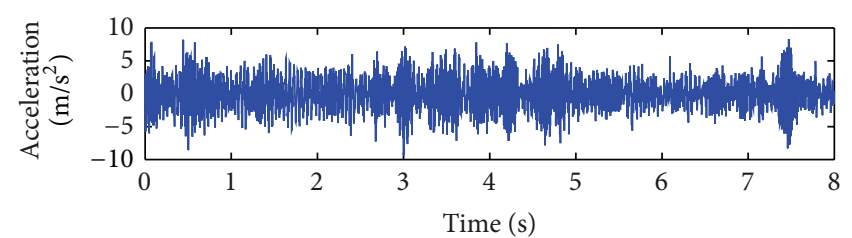

(b)

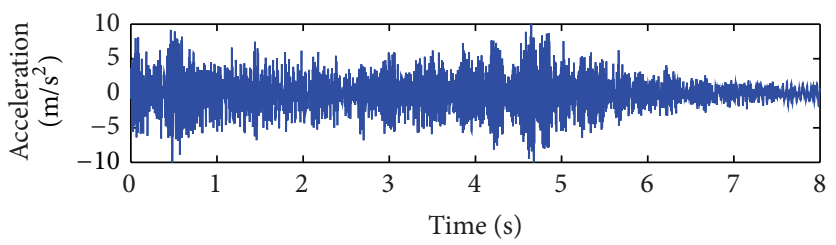

(d)

Figure 6: Acceleration responses of (a) Output 1, (b) Output 4, (c) Output 7, and (d) Output 11 of the time-varying structure.

the frequency domain and the power spectra (cross power spectra for Outputs 1-11 referred to Output 7), the modal parameters at time $t$ are estimated as shown in Figure 8.

7.6. Recursive Validation of the Estimated Modal Parameters. This section validates the estimated modal frequency as shown in Figure 8 using the recursive fuzzy clustering introduced in Section 5. The clustering results are shown in Figure 9 , in which the letters " $\mathrm{A}$ " and "B" denote the two clusters of the modal frequency, the color of these letters indicates the memberships of the modal frequency and the black dots show the prototypes of these clusters. The prototypes of the clusters move from left to right due to recursive behavior of the recursive fuzzy clustering algorithm. In this case, only the modal frequency is used for validation and the modal frequency before $0.5 \mathrm{~s}\left(t_{I}=0.5 \mathrm{~s}\right)$ is considered as the start data for initializing the fuzzy clustering. In the initialization phase, the parameters for fuzzy clustering are $M=100$ and $\zeta=10^{-5}$.

As shown in Figure 9, modal parameters are clustered and the memberships generally measure the quality of the corresponding modal parameters. The latter discriminates the low-quality estimates through weighting the data with the memberships in the next stage, as presented in (42).

7.7. Estimation of Continuous-Time Modal Parameters. Based on the validated and ordered modal parameters from the last stage, the continuous-time modal parameters are estimated by using the approach presented in Section 6 . The results of the continuous-time modal frequency and damping ratio are shown in Figures 10(a) and 10(b). In these recursive least squares, $L$ is 7 and $\kappa=5$.

As shown in Figure 10, the continuous-time estimation of the modal frequency coincides with the results of the baseline 


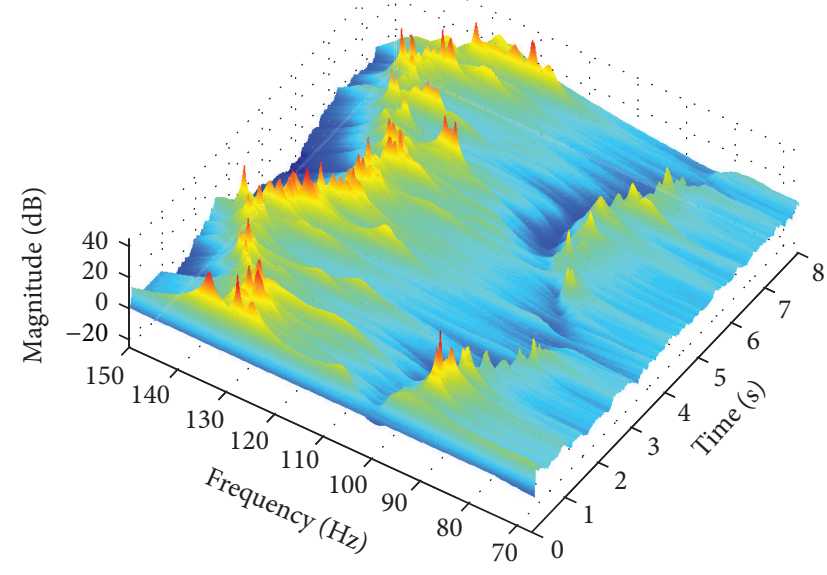

(a)

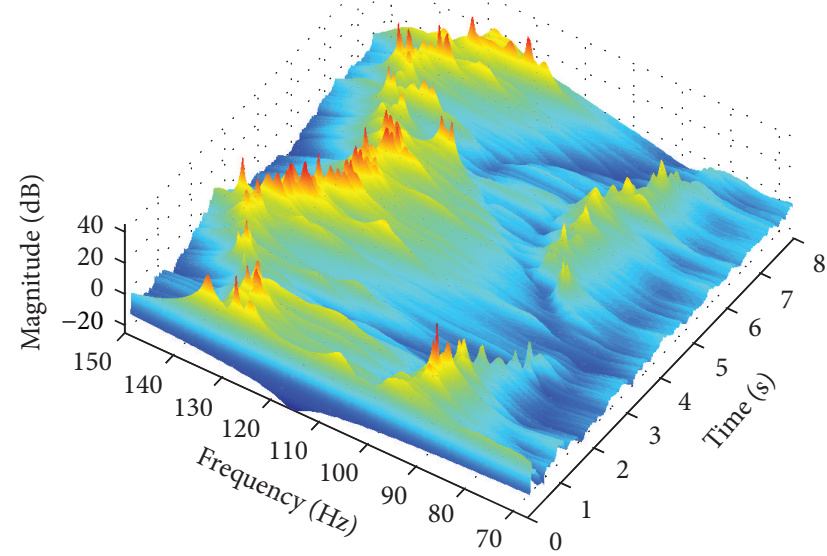

(c)

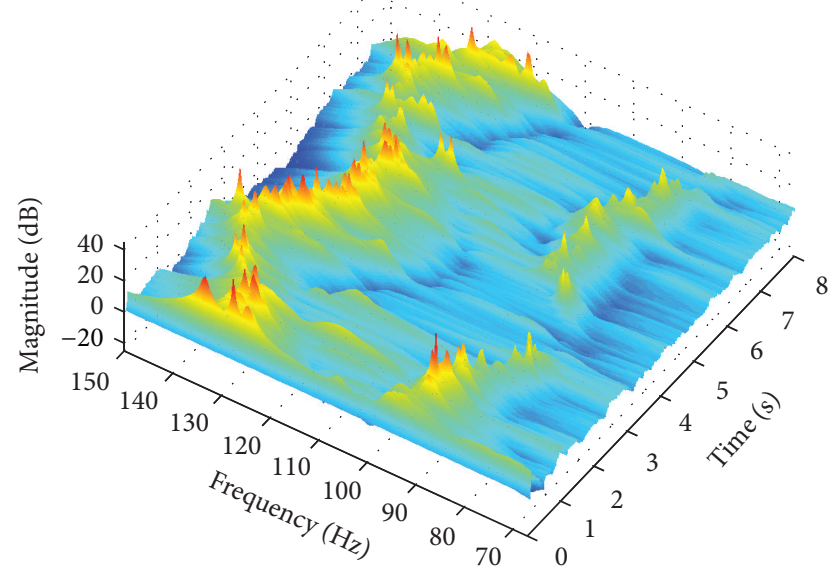

(b)

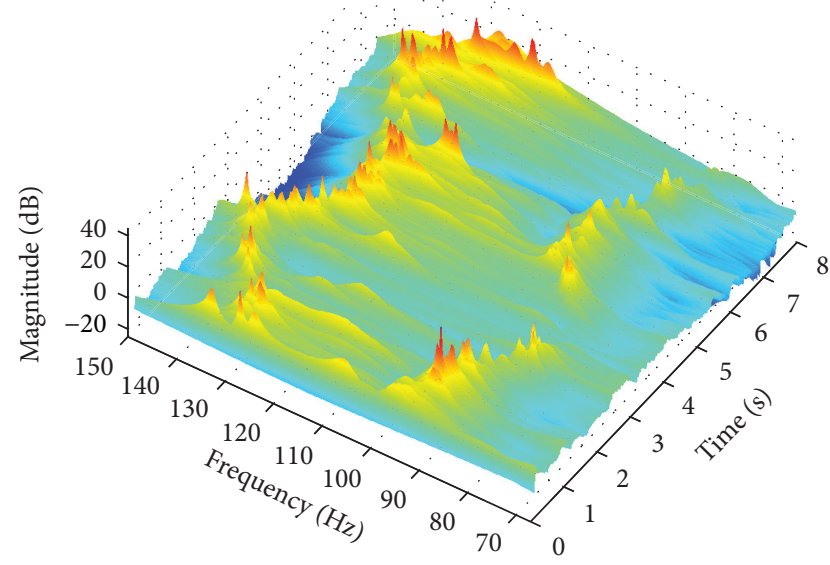

(d)

FIGURE 7: Cross power spectra for Outputs 1, 4, 7, and 11 referred to Output 7.

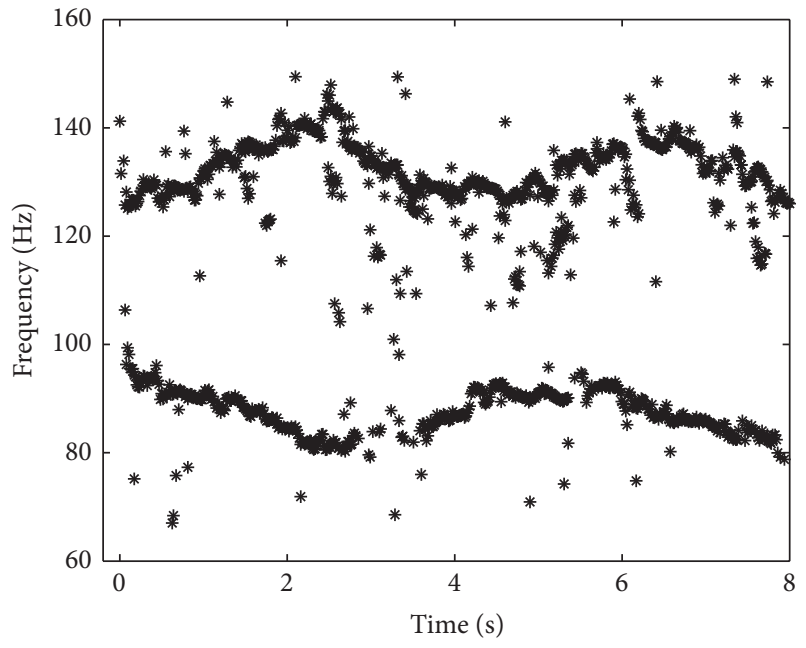

(a) Resonance frequency

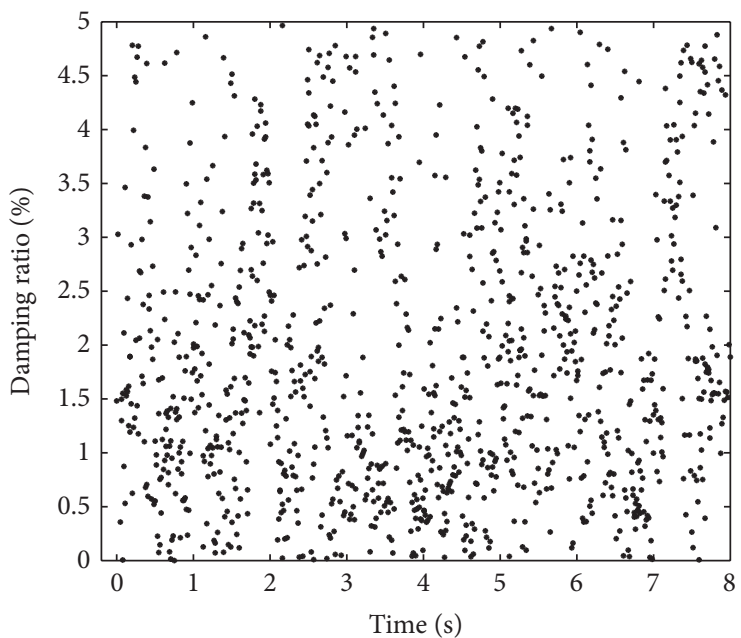

(b) Damping ratio

Figure 8: Estimated modal frequency and damping ratio. 


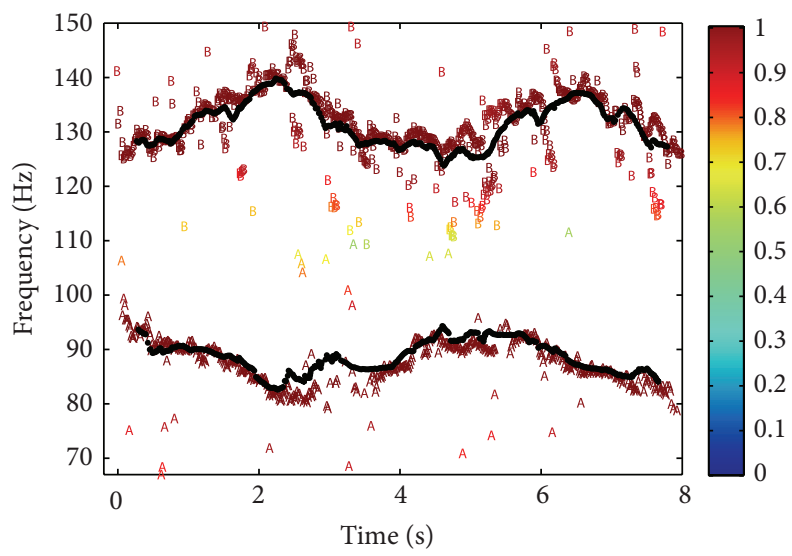

FIgURE 9: Recursive fuzzy clustering of the estimated modal parameters.

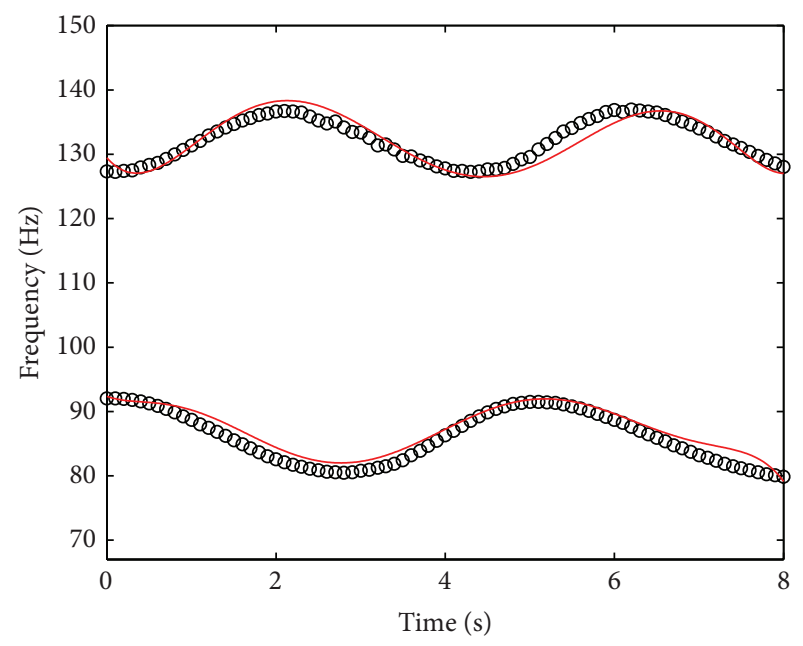

(a) Resonance frequency
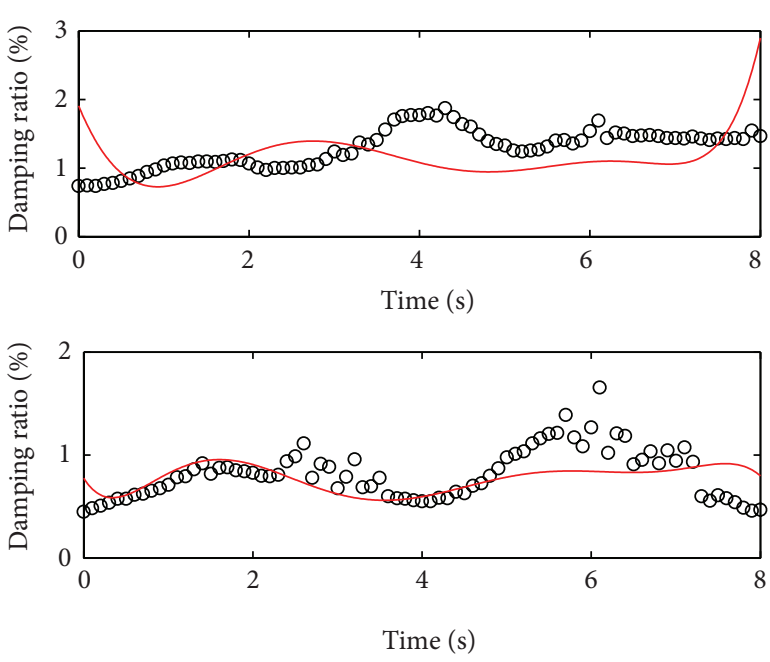

(b) Damping ratio

FIGURE 10: Estimated modal frequency and damping ratio.

models and that of the damping ratio is at the same level as the results of the baseline models.

\section{Conclusion}

This paper proposes a vector multistage recursive model parameter estimator for time-varying structures in the hybrid time and frequency domain. The research reproduces the available approaches of power spectra estimation, frequencydomain estimator for time-invariant structures, fuzzy clustering, and so forth, to present a recursive way of modal parameter estimation. This estimator has the capability of selecting the frequency band, which is helpful to achieve the parsimony of the estimation algorithm and may improve the accuracy and quality of the estimation in a limited frequency band. The recursive fuzzy clustering in the proposed estimator achieves the automatically recursive validation of the modal parameters at the current time instant. The final stage can obtain the continuous-time modal parameters through recursive least squares. The contribution of this paper is mainly the combination in the workflow itself and the necessary reproduction of each element in the proposed method.

In case the response data at time $t$ are measured, the modal parameters at that time instant are estimated, so it is recursive, which has the computational parsimony, and is potentially suitable for the real-time acquisition of the modal estimation for time-varying structures and is further possibly used in the applications such as the mode tracking, monitoring, and adaptive control of vibration.

\section{Conflict of Interests}

The authors declare that there is no conflict of interests regarding the publication of this paper.

\section{Acknowledgments}

The authors acknowledge the support for the work presented in this paper from the Beijing Institute of Technology 
Foundation for Basic Research (Grant no. 20120142009) and National Natural Science Foundation of China (Grant no. 11402022).

\section{References}

[1] K. A. Petsounis and S. D. Fassois, "Non-stationary functional series TARMA vibration modelling and analysis in a planar manipulator," Journal of Sound and Vibration, vol. 231, no. 5, pp. 1355-1376, 2000.

[2] K. A. Petsounis and S. D. Fassois, "Parametric time-domain methods for the identification of vibrating structures-a critical comparison and assessment," Mechanical Systems and Signal Processing, vol. 15, no. 6, pp. 1031-1060, 2001.

[3] A. G. Poulimenos and S. D. Fassois, "Parametric time-domain methods for non-stationary random vibration modelling and analysis-a critical survey and comparison," Mechanical Systems and Signal Processing, vol. 20, no. 4, pp. 763-816, 2006.

[4] A. G. Poulimenos and S. D. Fassois, "Output-only stochastic identification of a time-varying structure via functional series TARMA models," Mechanical Systems and Signal Processing, vol. 23, no. 4, pp. 1180-1204, 2009.

[5] M. D. Spiridonakos and S. D. Fassois, "Parametric identification of a time-varying structure based on vector vibration response measurements," Mechanical Systems and Signal Processing, vol. 23, no. 6, pp. 2029-2048, 2009.

[6] K. Liu, "Identification of linear time-varying systems," Journal of Sound and Vibration, vol. 206, no. 4, pp. 487-505, 1997.

[7] K. Liu, "Extension of modal analysis to linear time-varying systems," Journal of Sound and Vibration, vol. 226, no. 1, pp. 149$167,1999$.

[8] K. Liu and L. Deng, "Experimental verification of an algorithm for identification of linear time-varying systems," Journal of Sound and Vibration, vol. 279, no. 3-5, pp. 1170-1180, 2005.

[9] K. Liu and L. Deng, "Identification of pseudo-natural frequencies of an axially moving cantilever beam using a subspacebased algorithm," Mechanical Systems and Signal Processing, vol. 20, no. 1, pp. 94-113, 2006.

[10] R. Ghanem and F. Romeo, "A wavelet-based approach for the identification of linear time-varying dynamical systems," Journal of Sound and Vibration, vol. 234, no. 4, pp. 555-576, 2000.

[11] A. Roshan-Ghias, M. B. Shamsollahi, M. Mobed, and M. Behzad, "Estimation of modal parameters using bilinear joint time-frequency distributions," Mechanical Systems and Signal Processing, vol. 21, no. 5, pp. 2125-2136, 2007.

[12] X. Xu, Z. Zhang, H. Hua, and Z. Chen, "Identification of time-varying modal parameters using linear time-frequency representation," Chinese Journal of Mechanical Engineering, vol. 16, no. 4, pp. 445-448, 2003.

[13] E. Lourens, E. Reynders, G. De Roeck, G. Degrande, and G. Lombaert, "An augmented Kalman filter for force identification in structural dynamics," Mechanical Systems and Signal Processing, vol. 27, no. 1, pp. 446-460, 2012.

[14] S.-B. Song and C.-M. Pei, "A recursive method of timefrequency analysis for the signal processing of flutter test with progression variable speed," Chinese Journal of Aeronautics, vol. 18, no. 3, pp. 213-217, 2005.

[15] L. Ljung, System Identification: Theory for the User, Prentice Hall, Upper Saddle River, NJ, USA, 1999.
[16] N. Calinoiu, S. Bachir, and C. Duvanaud, "Mixed time and frequency-domain identification of radio frequency power amplifiers," in Proceedings of the 40th European Microwave Conference (EuMC '10), pp. 1018-1021, Paris, France, September 2010.

[17] S.-D. Zhou, W. Heylen, P. Sas, and L. Liu, "Parametric modal identification of time-varying structures and the validation approach of modal parameters," Mechanical Systems and Signal Processing, vol. 47, no. 1-2, pp. 94-119, 2014.

[18] H. van der Auweraer, P. Guillaume, P. Verboven, and S. Vanlanduit, "Application of a fast-stabilizing frequency domain parameter estimation method," Journal of Dynamic Systems, Measurement and Control, Transactions of the ASME, vol. 123, no. 4, pp. 651-658, 2001.

[19] H. van der Auweraer and B. Peeters, "Discriminating physical poles from mathematical poles in high order systems: use and automation of the stabilization diagram," in Proceedings of the 21st IEEE Instrumentation and Measurement Technology Conference (IMTC '04), vol. 3, pp. 2193-2198, Cornu, Italy, May 2004.

[20] G. C. Reinsel, Elements of Multivariate Time Series Analysis, Springer, Berlin, Germany, 1993.

[21] B. Cauberghe, P. Guillaume, P. Verboven, S. Vanlanduit, and E. Parloo, "On the influence of the parameter constraint on the stability of the poles and the discrimination capabilities of the stabilisation diagrams," Mechanical Systems and Signal Processing, vol. 19, no. 5, pp. 989-1014, 2005.

[22] F. Höppner, F. Klawonn, R. Kruse, and T. Runkler, Fuzzy Cluster Analysis: Methods for Classification, Data Analysis and Image Recognition, John Wiley \& Sons, 1999.

[23] D. Dovžan and I. Škrjanc, "Recursive fuzzy c-means clustering for recursive fuzzy identification of time-varying processes," ISA Transactions, vol. 50, no. 2, pp. 159-169, 2011.

[24] S. Van Huffel and J. Vandewalle, The Total Least Squares Problem: Computational Aspects and Analysis, Society for Industrial and Applied Mathematics, 1991.

[25] W. Heylen, S. Lammens, and P. Sas, Modal Analysis Theory and Testing, Katholieke Universiteit Leuven, Leuven, Belgium, 2007. 

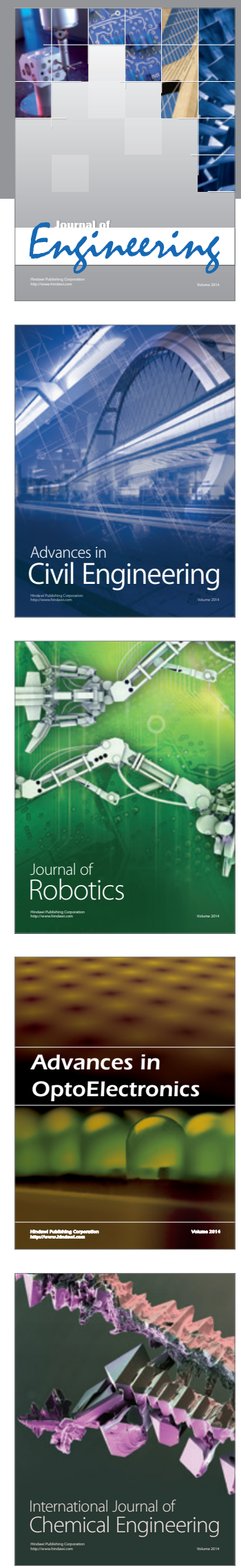

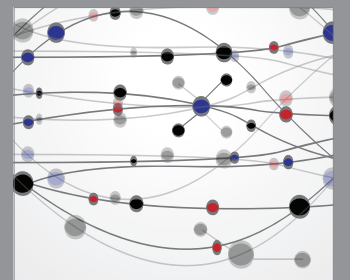

The Scientific World Journal
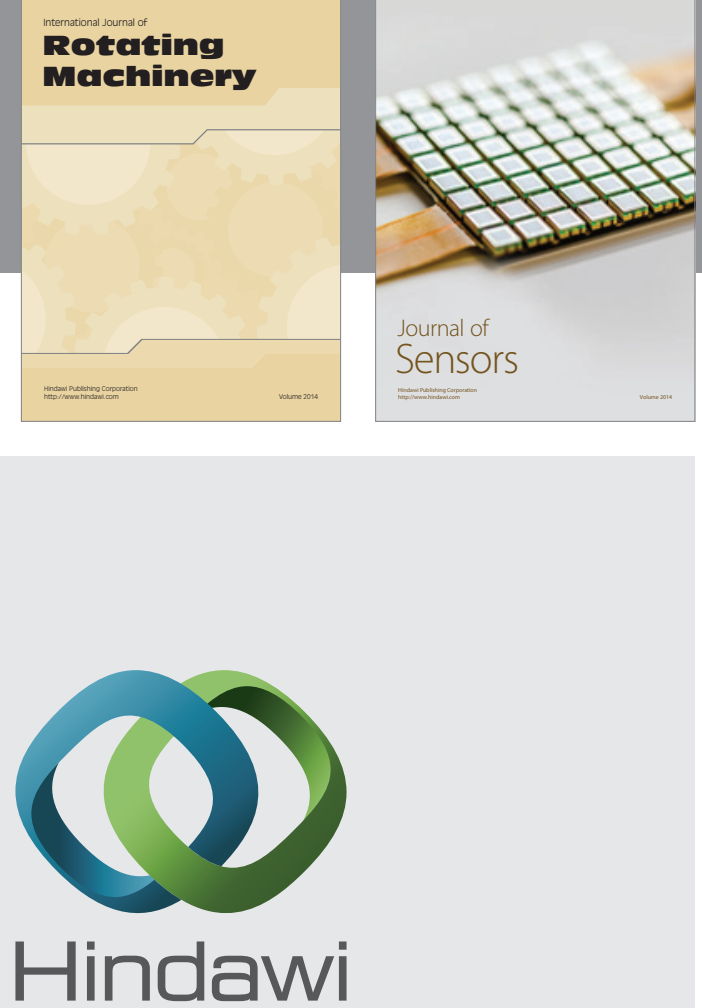

Submit your manuscripts at http://www.hindawi.com
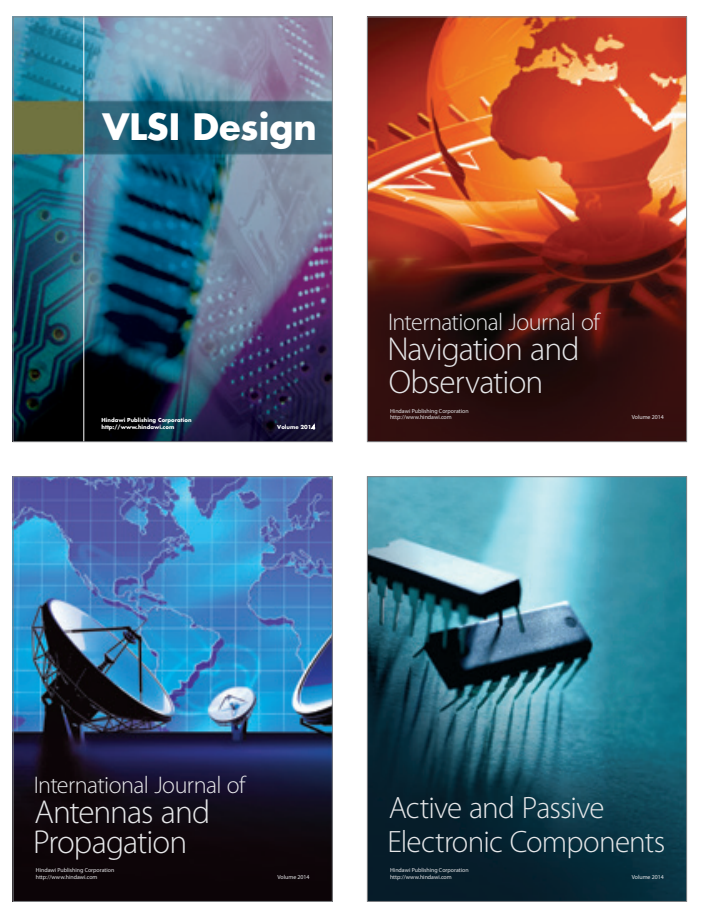
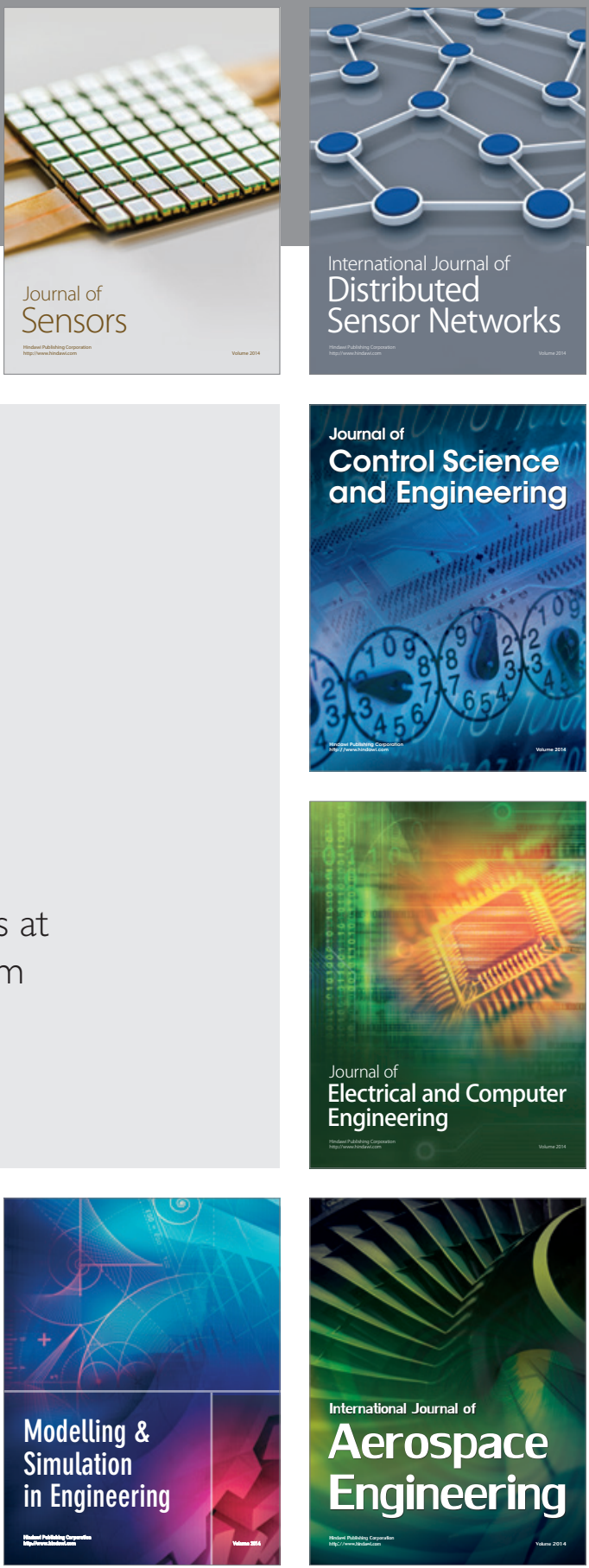

Journal of

Control Science

and Engineering
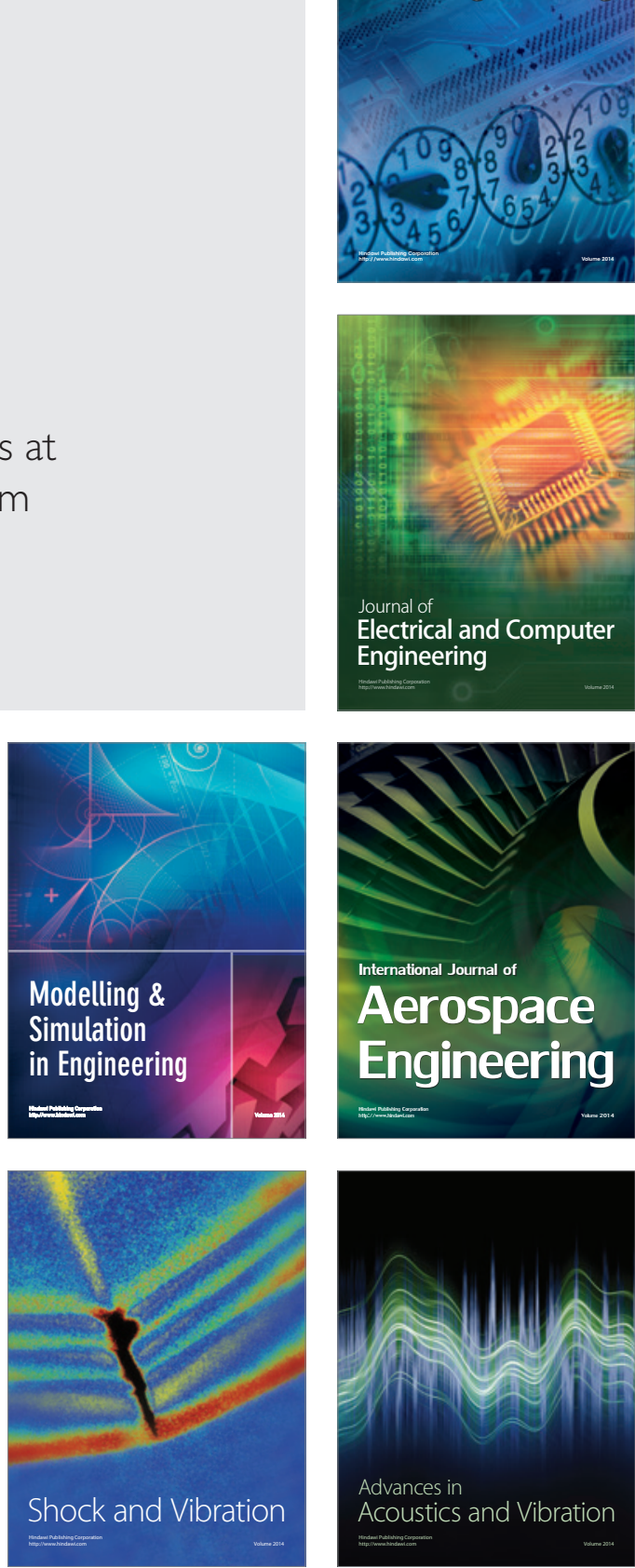